\title{
Utility of Hybrid Wireless Experimentation for Evaluation of Heterogeneous Wireless Architectures and Cross-Layer Protocols
}

\author{
Mahesh K. Marina ${ }^{a}$ \\ mmarina@inf.ed.ac.uk \\ Zhiguo Xu, Junlan Zhou and Rajive Bagrodia ${ }^{b}$ \\ \{zhiguo, zjl, rajive\}@cs.ucla.edu \\ ${ }^{a}$ School of Informatics, The University of Edinburgh, UK \\ ${ }^{b}$ Computer Science Department, University of California at Los Angeles, USA
}

\begin{abstract}
We consider hybrid wireless experimentation, which has emerged as an alternative and complementary methodology to physical experimentation and simulation for wireless network evaluation. Specifically, our focus in this paper is on WHYNET, a flexible hybrid evaluation framework providing the capability to seamlessly integrate simulated, emulated and physical networks, thereby enabling several ways of realizing a given target wireless network scenario each using physical (operational) and simulated elements in different combinations depending on the evaluation needs and available testbed resources. Using two novel and detailed case studies of WHYNET, we demonstrate the utility of the hybrid approach for realistic, scalable and cost-effective evaluation of heterogeneous wireless network scenarios and cross-layer protocol mechanisms.
\end{abstract}

\section{Introduction}

The availability of effective evaluation platforms is key to the development of high performance, reliable and energy efficient wireless network systems [1]. However, the design of such platforms is challenging as they need to satisfy a conflicting set of requirements - viz., realism, controllability, scalability and cost-effectiveness - arising from the need to support diverse experimentation needs and wide range of wireless networking and radio technologies. The two most common evaluation methodologies, physical experimentation and simulation, while offering a unique set of benefits, are insufficient in meeting those requirements. Physical experimentation is very realistic, but difficult to control and can be an expensive approach for evaluation of futuristic radio technologies and large-scale or mobile scenarios. Simulation, on the other hand, allows controlled and flexible experimentation of large-scale wireless network scenarios, but usually at the expense of realism.

In recent years, hybrid wireless experimentation has emerged as an alternative methodology complementary to physical experimentation and simulation for addressing the challenges associated with effective wireless network evaluation. Though the phrase "hybrid wireless experimentation" in the broadest sense encompasses various forms of emulation $[2,3,4,5$, $6,7,8]$, our focus in this paper is in the context of WHYNET evaluation framework [9]. WHYNET based on TWINE [10], MAYA [11] and SenQ [12] is a flexible evaluation framework that provides the capability to seamlessly integrate simulated, emulated and physical networks, thereby enabling several ways of realizing a given target wireless network scenario each using physical (operational) and simulated elements in different combinations depending on the evaluation needs and available testbed resources.

In this paper, we present two novel case studies for hybrid wireless experimentation in general and WHYNET evaluation framework in particular. These case studies demonstrate capabilities of the WHYNET framework in supporting realistic and scalable evaluation of wireless network scenarios involving diverse radio technologies and scenarios using cross-layer mechanisms. In particular, they highlight: (i) combined use of physical, simulation and emulation testbeds in realizing a large-scale heterogenous wireless network scenario spanning multiple radio technologies; (ii) the ability of WHYNET emulation facility to incorporate real-world interactions among protocol layers and the operating system for accurate evaluation of cross-layer protocol mechanisms. As we will elaborate in the next section, both these case studies are different yet complementary from existing studies in the literature focusing on hybrid wireless experimentation with the WHYNET framework $[10,9]$ in terms of capabilities demonstrated as well as application scenarios.

The remainder of this paper is structured as follows. Next section provides relevant background and discusses related work on case studies of hybrid wireless 
experimentation with the WHYNET framework. We will present two new case studies, the core of this paper, in Section 3 and conclude the paper in Section 4.

\section{Background and Related Work}

Physical experimentation and simulation are the two common evaluation methods for wireless networks. Physical experimentation with real wireless systems and channels (typically using small to medium scale testbeds) is invaluable for characterization of various real-world aspects of wireless networks (e.g., channel, usage patterns, traffic, mobility) and validation of research ideas in real-world settings. In recent past, several physical wireless network testbeds have been used for real-world protocol evaluations. These include MANET testbeds at CMU and Uppsala for ad-hoc routing protocol studies, and MIT Roofnet to evaluate mesh network performance (see [13] and references therein). This approach can be expensive for evaluation of large-scale or mobile scenarios and those involving emerging radio technologies (e.g., flexible and high-performance MIMO radios). Moreover, the inherent difficulty of controlling wireless channel behavior limits this approach in terms of experimental control to support repeatable experiments under diverse channel conditions, an important requirement for comprehensive and fair evaluation of cross-layer techniques; this problem becomes more severe as the target wireless network gets larger and more heterogeneous.

Simulation, on the other hand, is an alternative and widely used approach that supports flexible and controlled experimentation of arbitrary wireless network scenarios. It is especially useful for gaining insight into the efficacy of design alternatives at early stage of research involving new networking and radio technologies (ahead of their implementation in real systems) as well as studying the impact of scaling to larger and more stressful configurations, all in a costeffective manner. Several wireless network simulators exist (e.g., ns-2, GloMoSim, QualNet, OPNET) and are commonly used for wireless network evaluations. On the downside, this approach faces a tough balancing act between accuracy of models via minimal assumptions and abstractions, on one hand, and lower execution times and modeling related costs on the other; this limits its use for realistically studying system-wide interactions among real applications, operating system, hardware and channel dynamics.

The fact that neither physical experimentation nor simulation is sufficient to meet the requirements of an ideal evaluation methodology mentioned at the outset has motivated the work on alternative approaches for wireless network evaluation, which can be broadly said to fall under the "hybrid wireless experimentation" approach. This includes the work on various forms of emulation. Emulation is an intermediate approach between physical experimentation and simulation in that it uses real (physical) components interfaced with virtual (simulated) components to realize a target wireless network scenario. Among existing wireless network emulators, some emulate only the wireless channel $[5,7]$ whereas others also emulate the radio device [3, 4].

In an attempt to address the experiment control (including repeatability) and manageability issues associated with full-scale physical testbeds, some testbed research efforts "emulate" wireless channels while still using real radio devices. These efforts follow one of two approaches. In the first approach, which we refer to as scaled testbeds (e.g., ORBIT [7], MiNT [8]), the idea is to attenuate radio signals to restrict the range of communication to a smaller space, thus scaling the environment for over-the-air tests. Realism, repeatability (especially in presence of external uncontrolled sources of noise or interference) and the ability to support diverse experimental conditions of testbeds following this approach are not yet wellestablished and remains an active area of research. On the other hand, hardware-based channel emulators $[5,6]$ are highly realistic due to their detailed signal-level emulation of the wireless channel, while at the same time offer high degree of control in terms of experimenting with a wide range of channel conditions in a repeatable manner. On the downside, these hardware channel emulators have high cost and limited scale.

Other research efforts on wireless network emulators take a different approach by emulating wireless device and channel behaviors in software for increased flexibility and scalability at low cost. Earlier work in this category uses statistical (e.g., NIST Net [14]) or empirically derived models (e.g., trace modulation [2]) to subject higher layer protocols to coarse-grain wireless network dynamics in terms of delay and loss behavior. These emulators fail to realistically capture key wireless channel effects such as interference or cannot support experimentation under diverse channel conditions. Other emulators such as MobiEmu [15] emulate only node mobility (based on trace) and are primarily meant for testing ad-hoc routing protocol implementations. More recent work ad- 
ditionally models radio (MAC/PHY) and channel behaviors. MobiNet/ModelNet [4] adopts a centralized emulation approach in which routing, MAC/PHY, channel and node mobility are emulated separately from the emulation hosts on a workstation cluster. Additional processing and propagation delays required to communicate between the emulation hosts and the cluster can prevent this approach from accurate and seamless emulation. Besides, MobiNet cannot leverage existing routing protocol implementations and requires re-implementing them specifically for the cluster nodes. EMPOWER [3], on the other hand, takes a distributed emulation approach using a collection of emulation nodes. Further, each of these emulation nodes can emulate multiple wireless nodes from the target scenario for scalability. However, EMPOWER does not model PHY and only has an approximate MAC (CSMA/CA) model.

\section{WHYNET}

As noted earlier, our focus on hybrid wireless experimentation is in the context of WHYNET evaluation framework [9]. WHYNET based on TWINE [10], MAYA [11] and SenQ [12] is a flexible evaluation framework that provides the capability to seamlessly integrate simulated, emulated and physical networks, thereby enabling several ways of realizing a given target wireless network scenario each using physical (operational) and simulated elements in different combinations depending on the evaluation needs and available testbed resources. For example, the scenario can be partitioned into interconnected subnets with each subnet mapped to an instance of either physical, simulation or emulation components. Alternatively, it can be fully realized using either a physical testbed or simulation; thus, both physical experimentation and simulation are supported as special cases in the WHYNET framework.

The emulation capability in WHYNET based on TWINE is conceptually similar to EMPOWER, but can emulate radio and channel with high fidelity using detailed models in real time. Furthermore, the WHYNET framework supports seamless integration of emulated subnets with simulation and physical testbeds for greater scalability or realism. WHYNET as a whole is conceptually similar to Netbed (a successor of Emulab) [16] in that both aim to integrate simulation, emulation and physical experimentation within a single framework; the latter however is targeted towards wired network experimentation. Interested reader is referred to [9] and references therein for further details on the implementation of the WHYNET framework.

Several case studies of hybrid wireless experimentation with the WHYNET framework exist $[10,9]$. The uniqueness of the case studies presented in this paper lies in considering different usage modes from those in the past and focusing on newer target wireless network scenarios, thereby broadening the applicability of hybrid wireless experimentation. Two case studies are presented in [10] with combination of simulation and emulation as the hybrid evaluation mode in both cases. One study focuses on performance of diverse applications with distributed quality of service (QoS) mechanisms and highlights the need to account for application QoS requirements. Another study evaluates adaptive video streaming applications in large scale mobile networks and shows lack of correlation between network-centric metrics and user perceived performance, thus motivating the need for perceptual evaluation.

[9] presents three additional case studies two of which focus on heterogeneous wireless network scenarios. In one case, the performance of streaming video application in a heterogeneous vehicular Internet access scenario is studied where the scenario is realized using a combination of physical, simulated and emulated subnets with physical component corresponding to the wired Internet segment. We also consider a large-scale heterogenous wireless network scenario in one of our case studies, but our scenario involves multiple radio technologies with one of them realized using a physical testbed for which we do not have access to an accurate model to allow the use of simulation or emulation; thus we realize different radio technologies and bands using a combination of physical, simulation and emulation components. Another heterogenous wireless network scenario is studied in [9] with sensor and data mule nodes forming a mobile ad hoc network and realized using emulation and simulation. The third case study in [9] considers the performance of a standard mobile ad hoc network protocol and highlights the impact of interactions between protocols and other components of system software that may not be typically modeled in simulators. We also make similar observations, but in the context of cross-layer protocol mechanisms.

\section{Case Studies}

In this section, we present two novel case studies of hybrid wireless experimentation using the WHYNET evaluation framework. The first study focuses on a heterogeneous wireless network scenario spanning 
multiple radio technologies while the second involves cross-layer protocols. In the following we describe these studies in detail including how the network scenario is realized using WHYNET testbed components in each case and the resulting insights.

\section{III.A. Heterogeneous Wireless Network Architectures}

In this study, we evaluate a heterogeneous wireless network architecture involving multiple radio technologies using the WHYNET framework. In particular, we explore the use of cellular networks in conjunction with 802.11-based mesh networks for Internet access. Cellular networks, despite their wider coverage and good support for mobile voice applications, have lagged until recently in providing high data rates needed for Internet applications. This is changing with the roll out of $3 \mathrm{G}$ wireless data services such as CDMA-1xEVDO that offer peak rates around $2 \mathrm{Mbps}$ (comparable to wired broadband Internet access solutions such as DSL and CATV) and future enhancements promise much higher data rates (up to $46 \mathrm{Mbps}$ ). On the other hand, the success of 802.11 technology for indoor wireless LANs has led to its use in newer scenarios, notably community wireless mesh networks for wider and low-cost Internet access. In a typical mesh network, a set of access routers form a multihop backhaul network; a subset of these access routers with a wired Internet connection (e.g., $\mathrm{T}-1$, DSL, CATV) act as gateways to provide Internet access to mobile clients associated with any of the access routers. The use of dedicated wired T-1/T-3 lines for Internet connectivity in a mesh network is expensive, whereas other wired broadband access solutions such as DSL and CATV are limited to highly populated metropolitan areas, also due to economic reasons. As an alternative access approach, the use of wireless wide-area network (WWAN) or cellular links offers a low-cost solution for ubiquitous broadband Internet connectivity by leveraging vast amount of extant cellular infrastructure. Also, the synergistic use of both cellular and 802.11 networks can better meet the future needs for increased performance and coverage as future advances in both cases are driven by a common set of emerging technologies (e.g., dynamic spectrum access, smart antennas) with the potential to outpace wired alternatives.

We therefore consider a heterogeneous wireless mesh network architecture with some dual-mode access routers (equipped with both 802.11 and $3 \mathrm{G}$ cellular interfaces) serving as Internet gateways, whereas the rest of them single-mode with only a 802.11 in-

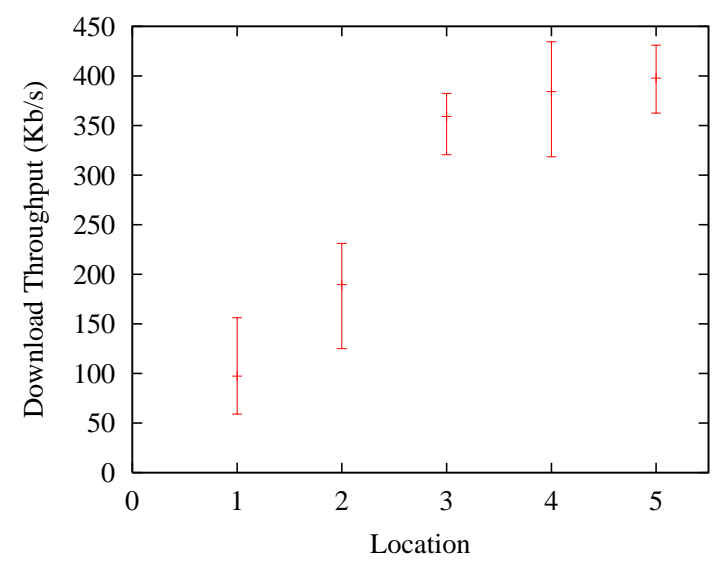

Figure 1: Spatio-temporal behavior of CDMA1xEVDO link performance based on measurements.

terface. This architecture can be seen as a generalization of the two-hop-relay architecture in [17]; it differs from other architectures based on heterogeneous radio technologies such as UCAN [18] in the use of fixed relay nodes (access routers) of which not all of them need to be dual-mode.

Our goal is to evaluate Internet access performance with the above architecture as perceived by a user at a wireless host attached to the mesh network as a function of number of Internet (WWAN) gateways and their relative physical placement. Note that these two factors influence the characteristics of the multihop path in the mesh network between the wireless host and the gateway node, as well as the cellular link connecting the gateway to the Internet; these characteristics together determine end-to-end performance.

Below we describe how we map the above experimental scenario to the hybrid testbed components. To this end, we begin with a discussion on the measured performance behavior of cellular links.

Cellular links can exhibit high spatio-temporal variations in terms of bandwidth, latency and loss characteristics even with stationary nodes. A number of factors contribute to these variations including: long distance links, terrain, larger delay spread due to multipath fading, environmental mobility effects, interference among multiple users, link adaptation and scheduling mechanisms used at the base station. As shown in Figure 1, the above assertion is supported by our measurements of download speeds using a commercial wireless broadband access service based on CDMA-1xEVDO technology. These measurements were taken at nearby indoor/outdoor locations around Boelter Hall at UCLA. The data for each location cor- 


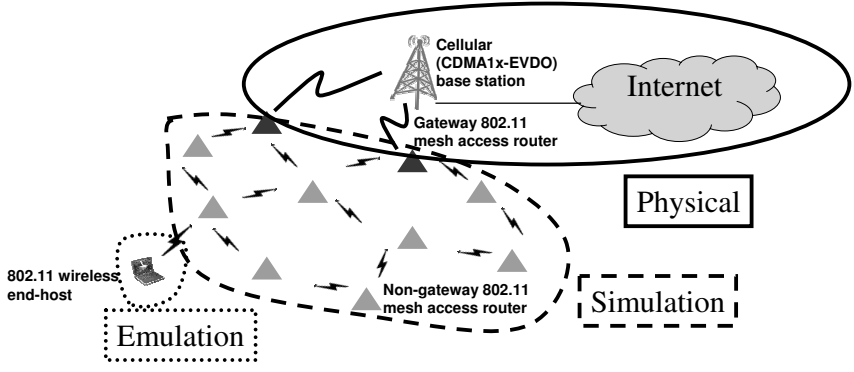

Figure 2: Realization of an integrated cellular/mesh network scenario using WHYNET evaluation framework and physical cellular (CDMA-1xEVDO) links.

responds to five back-to-back measurements for $1 \mathrm{MB}$ image download from the web. Since we obtained this data during early hours of the morning, the effect of interference may not be significant. We can make several observations based on this data. First, download speeds at different locations can be significantly different (up to factor of four). Second, short-term variations are considerable at locations with poor link performance. Third, the measured average throughput is at most around $400 \mathrm{Kbps}$, near the lower end of the range advertised by the service provider. We have also observed similar behavior as above from the measurements using the CDMA2000-1X base station deployed at UCSD as part of the WHYNET testbed infrastructure.

The preceding discussion points to the difficulty in modeling of the cellular link behavior for realistic experimentation, whereas accurate radio/channel models for $3 \mathrm{G}$ cellular links are not readily available for use in wireless network evaluations. Since we would like to realistically capture real-world behavior of cellular link characteristics in our evaluation without incurring the high modeling costs, we use physical mode of experimentation for the cellular link part of the scenario. Mesh networks, on the other hand, are larger in scale with typical deployments ranging from several tens to a hundred nodes. In addition, mesh network links tend to be relatively stable due to dense deployments and detailed models for such links are widely available. Given that this is a feasibility study

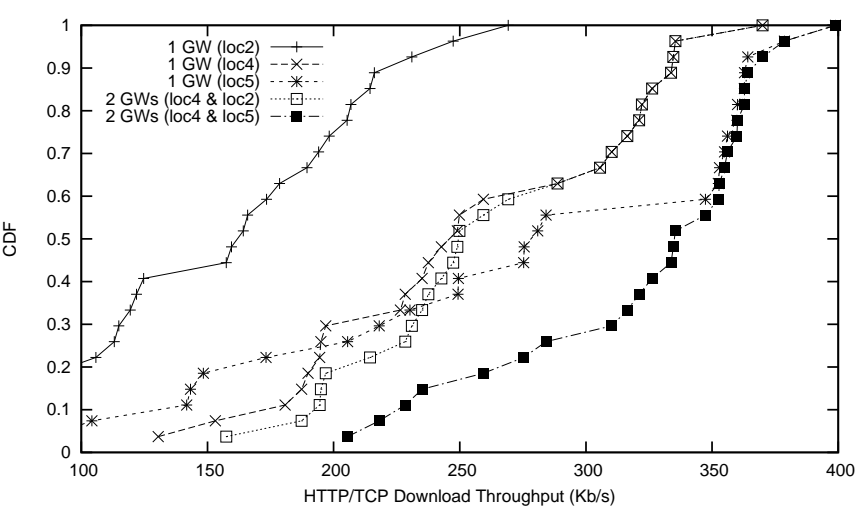

Figure 3: Impact of Internet gateways and their placement on user-perceived download performance in the integrated cellular/mesh architecture.

with focus centered around the effects of using cellular gateways, we find it convenient to use simulation ${ }^{1}$ to represent the mesh network of access routers with adequate fidelity. This choice avoids the high cost and tedious setup issues associated with physical experimentation of large scale scenarios, while providing adequate fidelity. Moreover, it allows us to more easily identify the effect of cellular link dynamics and to study alternative routing/gateway selection strategies. Finally, the 802.11 host attached to the mesh network in our scenario is emulated to experiment with real applications. In our study, we assume that the host is stationary and use web (HTTP) traffic as the application. However, it is quite straightforward to extend the evaluation to other application scenarios such as mobile clients running voice over IP (VoIP). Figure 2 illustrates the realization of above scenario using a combination of emulation, simulation and physical testbeds that are integrated using the WHYNET framework leveraging TWINE.

Figure 3 shows the results obtained from using the hybrid testbed as described above to realize a heterogeneous 802.11 mesh and cellular network scenario. The scenario consists of one wireless host and a 30 node $802.11 \mathrm{~b}$ mesh network with either 1 or 2 gateway nodes at different randomly chosen locations. In Figure 3, the impact of number of gateways and their placement is shown as a CDF of average throughputs (for download of a $2 \mathrm{MB}$ image from the Internet) observed from a host associated with every nongateway router in the mesh network. The locations for the gateway nodes indicated in the legend corre-

\footnotetext{
${ }^{1}$ Alternatively, access routers in the mesh network can be emulated when testing with real implementations of routing protocols prior to their use in a real deployment.
} 
spond to the locations shown in Figure 1. In fact, the measurements for each location in Figure 3 were taken immediately after the corresponding measurements in Figure 1. The staircase pattern of the curves for single gateway case indicates the performance observed at various points in the mesh network differing in their distance (hops) to the gateway node - performance degrades with increasing hops because of greater inter-hop interference and higher likelihood of channel-related losses with longer paths. The curves for the two gateway case are obtained by taking the best throughput observed with either gateway. These results clearly show that choice of locations for the gateway nodes has significant impact on performance because of the high spatial dependence of the cellular link performance. Moreover, judicious placement of multiple gateways can yield more uniform performance in the mesh network.

From an evaluation viewpoint, this study shows that hybrid approach (through combined use of physical, simulation and emulation components) can lead to useful insights when evaluating heterogeneous wireless scenarios, and that it is a realistic, scalable and cost-effective approach for evaluating such scenarios in a lab scale setting.

\section{III.B. Cross-Layer Protocols}

We now present a case study that evaluates a crosslayer protocol, specifically XCP [19], in a hybrid wired/wireless network scenario on the WHYNET testbed. XCP is a recently proposed Internet congestion control protocol that has received considerable attention in the research community. XCP adopts a cross-layer approach in that uses explicit, precise feedback from the network about the level of congestion and adapts the rate at the sender (in the transport layer) accordingly. This is in contrast to the end-to-end approach in TCP where the sender probes for available bandwidth by gradually increasing the sending rate and infers congestion implicitly via packet loss. A particular capability of XCP makes it well-suited for the wireless environment even though it was originally intended as an efficient alternative to TCP over high bandwidth-delay product networks (e.g., high-speed optical networks, large delay satellite links). XCP enables identification of noncongestion related wireless losses through its ability to decouple rate control from error control via precise feedback. XCP, however, requires accurate available bandwidth estimation support at each node on the path between sender and receiver for accurate feedback calculation, a challenging issue over shared and lossy wireless channels. While underestimation of available bandwidth clearly leads to poor utilization of network capacity, the inflated feedback from overestimation can also create inefficiency by causing congestion and buffer overflows. Recent experimental work on XCP [20] has highlighted the negative impact of bandwidth estimation errors on XCP performance in the wired network context with shared Ethernet, whereas our focus is on the more complex wireless medium.

Our goal is to experimentally evaluate XCP's sensitivity to bandwidth estimation errors over wireless links. WHYNET emulator based on TWINE is an ideal evaluation environment for this study because it allows reproducible evaluations with diverse channel conditions (e.g., interference, channel related losses) while being realistic (as indicated by the validation results in [10]). Compared to channel emulators (e.g., $[5,6,7,8])$, it permits extending the evaluation to complex scenarios (e.g., larger number of wireless devices and multihop communication) as well as studying novel bandwidth estimation techniques that may require changes to the MAC protocol (usually implemented in the firmware with commodity radio devices). In this study, we focus on the popular 802.11based wireless LAN scenario. We use a Linux implementation of XCP from Zhang and Henderson [20]; this implementation is based on standard TCP implementation and uses TCP option fields to allow exchange of information such as congestion feedback between XCP end hosts and routers. Rather than use an actual bandwidth estimation capability, we run experiments over a wide range of static values for the "estimated bandwidth" to reflect a wide range of estimation errors (covering underestimation, accurate estimation and overestimation cases).

We first consider the simplest scenario of a single wireless link with no wireless losses. For this scenario, our testbed configuration consists of two laptops (Dell Latitude D600) running XCP over an emulated 802.11 link using WHYNET emulator. The MTU is set to 512 bytes and $802.11 \mathrm{~b}$ is used with PHY data rate fixed at $11 \mathrm{Mbps}$. For comparison, we also present ns-2 simulation results with identical settings with XCP model used in [19] (XCP model is currently available only in ns-2 simulator). Figure 4(a) and (b) show the throughput results obtained using $\mathrm{XCP}$ as a function of capacity estimate for a large file transfer (80MB) with WHYNET emulator and ns-2 respectively. TCP results are also included for reference. Note that there is a marked difference in behavior between the two set of results especially in the 


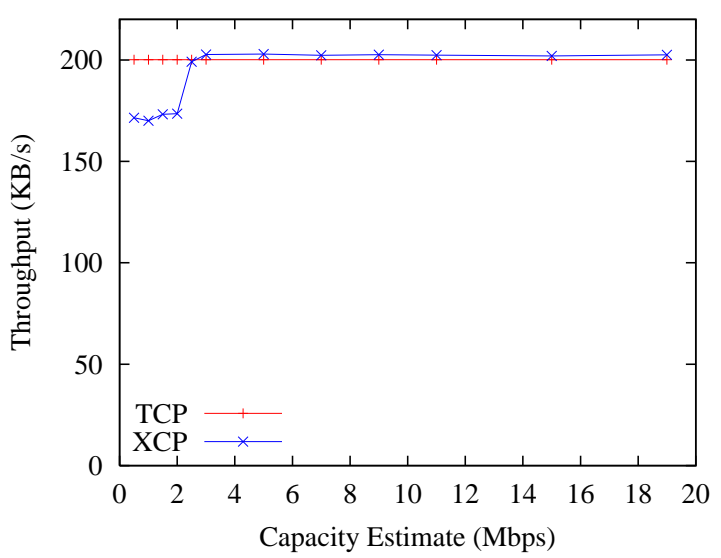

(a) WHYNET emulator

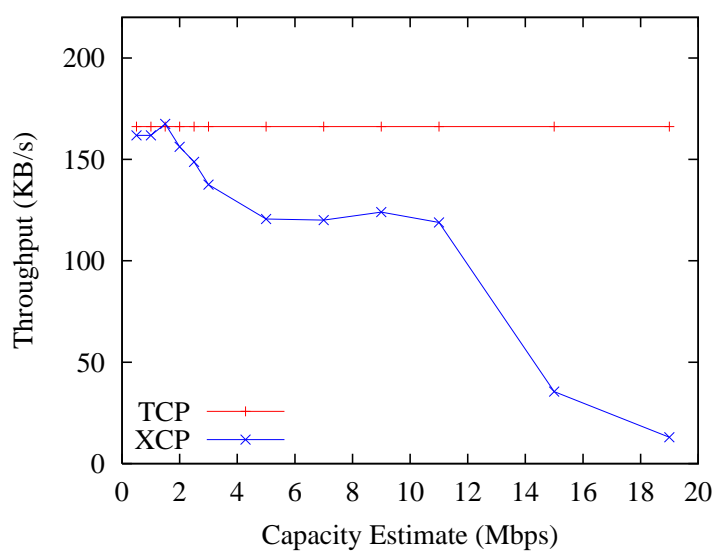

(b) ns-2 simulator

Figure 4: Impact of bandwidth estimation errors on XCP performance on a single wireless link using the WHYNET emulator and ns-2 simulator.

overestimation case. In the bandwidth overestimation case, the XCP router provides inflated feedback to the sender (both on the same node in this scenario), causing the latter to inject data at higher than optimal rate. With WHYNET emulator, bandwidth overestimation does not have any effect on XCP performance in this single link scenario because the sender host is stopped from sending more data beyond what can be handled by its device, thereby preventing any packet loss due to buffer overflow. We observed similar behavior when we turned off emulation and configured the hosts to use the built-in 802.11 interface instead. Results with ns-2, however, show that XCP performance degrades with increasing amounts of bandwidth overestimation. This we found was due to the modeling of interaction between network (IP) and link layers in the wireless protocol stack in ns-2 that differs from reality. Specifically, ns-2 allows packets to be transferred to the interface buffer (implemented as a droptail queue with a default size of 50 packets) regardless of the current buffer occupancy, which in turn causes IP layer to overflow the network interface and drop packets.

In our next experiment, we added a physical gigabit wired link to the scenario to create a typical wireless LAN scenario, where the wireless host connects to a wired backbone network via the AP. For this wired/wireless scenario, we consider file transfer from wireless host to the wired host via the AP ("upload") as well as in the reverse direction ("download"). Figure 5 shows the results obtained with WHYNET emulator for this scenario. Impact of bandwidth estimation in the upload case is similar to the previously discussed isolated wireless link scenario because the

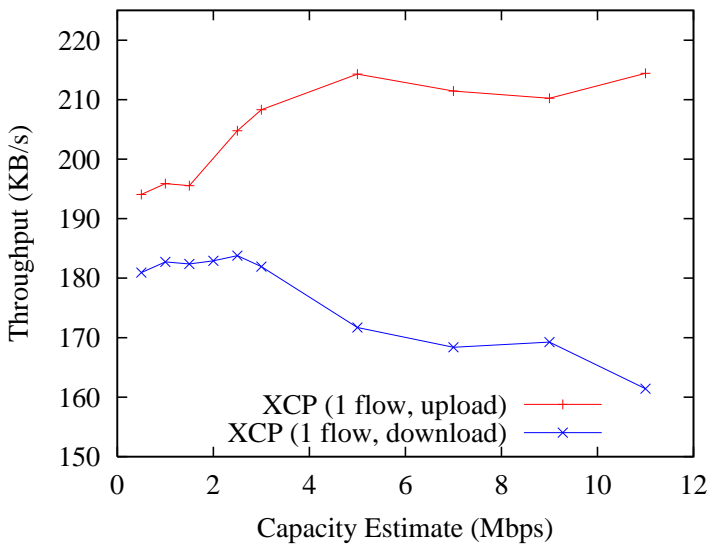

Figure 5: Impact of bandwidth estimation errors on XCP performance in a hybrid wired/wireless network scenario using the WHYNET emulator.

wireless hosts sends directly over the bottleneck wireless link (11Mbps). The download case behaves differently with overestimation hurting XCP throughput. This is because the bottleneck is now away from the sender (wired host) at the AP where incoming packets coming over the wired link can get dropped due to lack of buffers. Thus, the impact of bandwidth estimation errors on XCP performance depends on bottleneck location on the path.

The above results demonstrate the utility of WHYNET framework in facilitating realistic evaluations of cross-layer protocols by accurately capturing real-world interactions between different protocol layers. 


\section{Conclusion}

In this paper, we have considered hybrid wireless experimentation in the context of the recently proposed WHYNET evaluation framework. In particular, we have presented two novel case studies showcasing the utility of the hybrid approach and WHYNET framework for realistic and scalable evaluation of nextgeneration wireless network scenarios involving diverse radio technologies and cross-layer protocols. The presented case studies consider different hybrid wireless experimentation modes from those in the past and focus on newer target wireless network scenarios, thereby broadening the applicability of hybrid wireless experimentation.

\section{Acknowledgments}

This work has been funded by the NSF under the Network Research Testbed grant WHYNET: Scalable Testbed for Next Generation Mobile Wireless Networking Technologies (award number ANI-0335302). We thank Salih Ergut, Rajesh Mishra and Ozdemir Akin from UCSD for their help with EVDO cards, and Yongguang Zhang (HRL) and Prof. Medy Sanadidi (UCLA) for XCP Linux implementation and helpful discussions. We also thank Zhengrong Ji, Maneesh Varshney and Yi Yang for their helpful feedback and discussions.

\section{References}

[1] NSF Workshop on Network Research Testbeds. http://wwwnet.cs.umass.edu/testbed_workshop/, Oct 2002.

[2] B. D. Noble, M. Satyanarayanan, G. T. Nguyen, and R. H. Katz. Trace-Based Mobile Network Emulation. In Proc. ACM Sigcomm, 1997.

[3] P. Zheng and L. Ni. EMPOWER: A Network Emulator for Wireline and Wireless Networks. In Proc. IEEE Infocom, 2003.

[4] P. Mahadevan, A. Rodriguez, D. Becker, and A. Vahdat. MobiNet: A Scalable Emulation Infrastructure for Ad Hoc and Wireless Networks. In Proc. MobiSys 2005 Workshop on Wireless Traffic Measurements and Modeling (WiTMeMo), 2005.

[5] G. Judd and P. Steenkiste. Using Emulation to Understand and Improve Wireless Networks and Applications. In Proc. 2nd Symposium on Networked Systems Design \& Implementation (NSDI), 2005.

[6] PropSim. Propsim C8 - Wideband Multichannel Simulator. http://www.propsim.com/.

[7] D. Raychaudhuri et al. Overview of the ORBIT Radio Grid Testbed for Evaluation of NextGeneration Wireless Network Protocols. In Proc. WCNC, 2005.

[8] P. De, A. Raniwala, S. Sharma, and T. Chiueh. MiNT: A Miniaturized Network Testbed for Mobile Wireless Research. In Proc. IEEE Infocom, 2005.

[9] M. Varshney et al. WHYNET: A Framework for In-Situ Evaluation of Heterogeneous Mobile Wireless Systems. In Proc. ACM MobiCom 2007 Workshop on Wireless Network Testbeds, Experimental evaluation and CHaracterization (WiNTECH), 2007.

[10] J. Zhou, Z. Ji, and R. Bagrodia. TWINE: A Hybrid Emulation Testbed for Wireless Networks and Applications. In Proc. IEEE Infocom, 2006.

[11] J. Zhou, Z. Ji, M. Takai, and R. Bagrodia. Maya: Integrating hybrid network modeling to the physical world. ACM Transactions on Modeling and Computer Simulation, 14(2), 2004.

[12] M. Varshney, D. Xu, M. Srivastava, and R. Bagrodia. SenQ: A Scalable Simulation and Emulation Environment for Sensor Networks. In Proc. 6th International Conference on Information Processing in Sensor Networks (IPSN), 2007.

[13] P. De, A. Raniwala, S. Sharma, and T. Chiueh. Design Considerations for a Multi-hop Wireless Network Testbed. IEEE Communications Magazine, 43(10), 2005.

[14] M. Carson and D. Santay. NIST Net A Linuxbased Network Emulation Tool. ACM SIGCOMM Computer Communications Review, 33 (3), July 2003.

[15] Y. Zhang and W. Li. An Integrated Environment for Testing Mobile Ad-Hoc Networks. In Proc. ACM MobiHoc, 2002.

[16] B. White et al. An Integrated Experimental Environment for Distributed Systems and Networks. In Proc. OSDI, 2002. 
[17] H. Wei and R. Gitlin. Two-Hop-Relay Architecture for Next-Generation WWAN/WLAN Integration. IEEE Wireless Communications Magazine, 11(2), 2004.

[18] H. Luo et al. UCAN: A Unified Cellular and Ad-Hoc Network Architecture. In Proc. ACM MobiCom, 2003.

[19] D. Katabi, M. Handley, and C. Rohrs. Congestion Control for High Bandwidth-Delay Product Networks. In Proc. ACM Sigcomm, 2002.

[20] Y. Zhang and T. Henderson. An Implementation and Experimental Study of the eXplicit Control Protocol (XCP). In Proc. IEEE Infocom, 2005. 